\title{
Attend an open house on digital fabrication
}

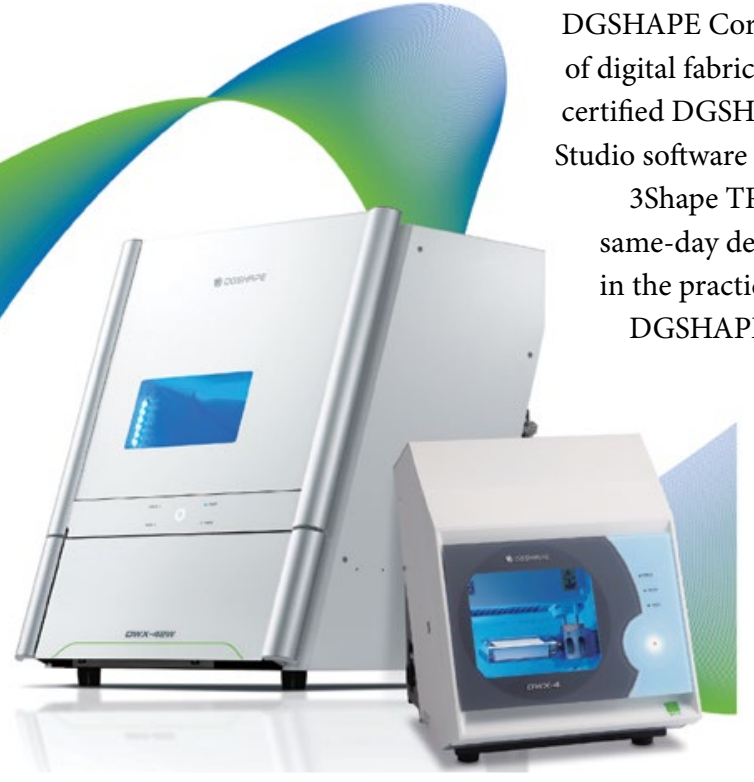

DGSHAPE Corporation, a wholly-owned subsidiary of Roland DG Corporation and a leading provider of digital fabrication tools, including 3D milling and engraving machines, has announced that 3Shape has certified DGSHAPE's DWX-4 and DWX-42W as Trusted Connection milling devices for their TRIOS Design software package.

TRIOS Design Studio software enables dental professionals to go beyond scanning with ay designing and milling of crowns, inlay/onlays, bridges, veneers, and screw-retained crowns right he practice. 3Shape Trusted Connection milling machines are tested avnd validated by the company.

GSHAPE's DWX-42W and DWX-4 represent the best solutions for a dental clinic to operate an in-office laboratory and get started with CAD/CAM workflows for same-day and next-day dentistry. Both machines offer single touch operation and are engineered from the ground up with in-house spindles and simple maintenance procedures. Known for their legendary reliability, DWX devices are praised by their owners for their ability to run unattended and provide excellent milling quality in the margins as well as occlusion.

Join the Roland DGSHAPE team and ESM for their 3SHAPE open house event on Wednesday 27 November 2019 in Clevedon, Somerset. For more information, or to confirm your attendance to this free event call 01275335540 or email Joe Dickinson jdickinson@ rolanddg.com.

www.dgshape.com

\section{The perfect platform for the expanded range}

For dentists looking for ideas and inspiration, the BDIA Dental Showcase in October was the place to be.

For the first time, the stand not only featured COLTENE materials, but the additional manufacturing of Micro-Mega, the French endodontic company, plus SciCan, the Canadian equipment manufacturer of autoclaves and washer-disinfectors. The show was the perfect platform to introduce the expanded range under the COLTENE brand.

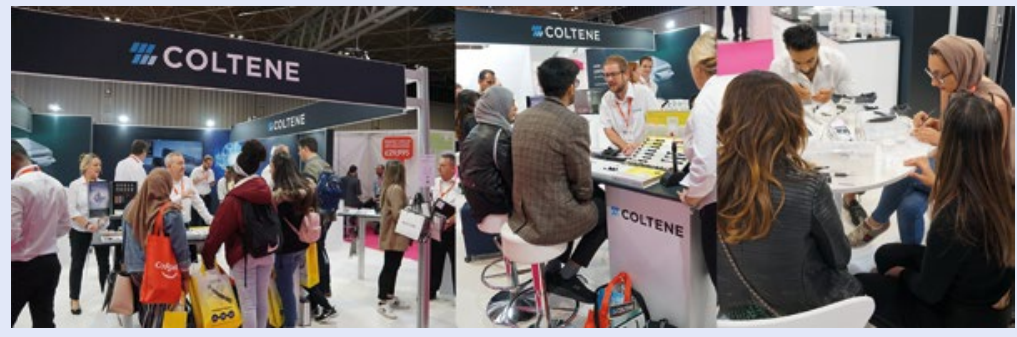

From general dentisty to restorative endodontic cases,

COLTENE has a solution. This year saw two key product launches. The HyFlex EDM Glidepath file 15/.03 for curved and narrow canals and COLTENE also now offers BRILLIANT COMPONEER for chairside restorations.

If you did not get to the event, visit the website or call, to discuss how COLTENE could upgrade your dentistry. Visit www.coltene.com, email info.uk@coltene.com or call 01444235486.

\section{Latest generation rubber interdental brushes}

Generating considerable interest at the BDIA Showcase this year, delegates were delighted to discover the brand new Clean Between Pro Interdental Brushes from Wisdom Toothbrushes, a company renowned for its comprehensive range of oral healthcare products.

The latest generation rubber interdental brushes now feature a uniquely curved profile, a broad thumbgrip, a reinforced stem and a robust, brush-like tip. These improved features are designed to deliver a gentle yet effective clean with better access to all areas of the mouth and a longer-lasting product.

Clinically proven to reduce plaque and bleeding, the Wisdom Clean Between Pro Interdental Brushes come in packs of 30 and in three sizes - small, medium and large. They also have a detachable transparent case for simple storage and for patients on the go.

Visit www.wisdomtoothbrushes.com or call 01440714800.

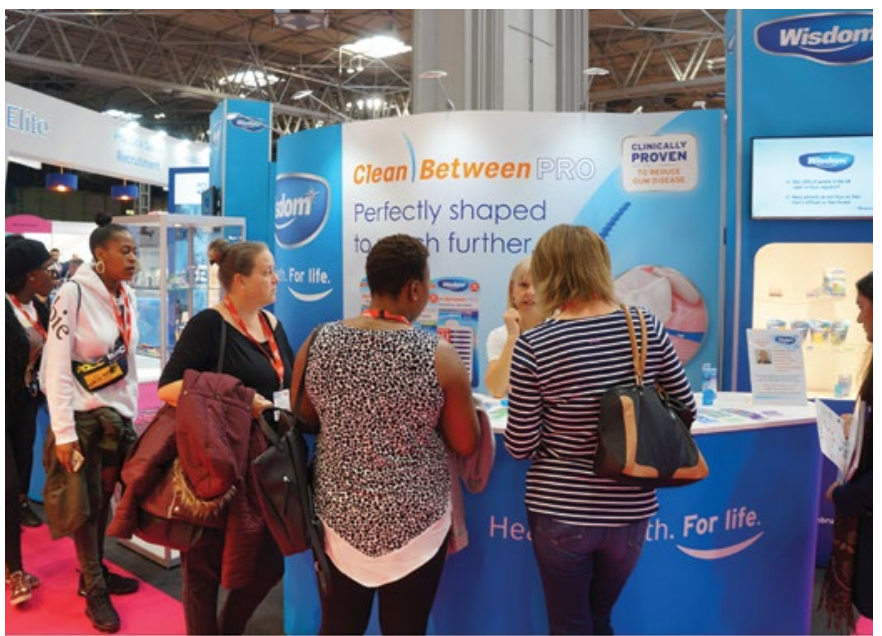

